\section{Sur les systèmes d'équations différentielles dont toutes les solutions sont bornées}

\author{
par Z. SzMydx (Kraków)
}

Considérons le système d'équations différentielles

(1)

$$
x^{\prime}=A x+f(t, x),
$$

où $A$ désigne une matrice constante,

$$
\left.\|f(t, x)\| \leqslant g(t)\|x\|^{1}\right), \quad \int_{0}^{\infty} g(t) d t<\infty
$$

et toutes les solutions du système $y^{\prime}=A y$ sont bornées pour $t \geqslant 0$.

H. Weyl [1] a démontré que toutes les solutions du système (1) sont bornées. R. E. Winograd [2] a construit un exemple ${ }^{2}$ ) montrant que cette propriété n'a pas lieu pour certains systèmes

$$
x^{\prime}=A(t) x+f(t, x)
$$

dont les matrices $A$ dépendent de la variable $t$. De plus Winograd a dé montré que chaque solution du système (3) est bornée dans les hypothòses suivantes:

Нүротніs: 1. La norme de la matrice fondamentale $Y(t)$ du système

$$
y^{\prime}=A(t) y
$$

est bornée, c'est-d̀-dire il existe un nombre $M<\infty$ tel que

$$
\left.\|Y(t)\| \leqslant M \quad \text { pour } \quad 0 \leqslant t<\infty^{3}\right) .
$$

1) Les normes du vecteur $w=\left(w_{1}, \ldots, w_{n}\right)$ et de la matrice $B=\left(b_{k j}\right)$ sont donnéses respectivement par les formules

$$
\|w\|^{2}=\sum_{i=1}^{n}\left|w_{i}\right|^{2} \quad \text { et } \quad\|B\|^{2}=\sum_{i, j=1}^{n}\left|b_{i j}\right|^{2} .
$$

2) Dans cet exemple on $a: A(t)=\left(\begin{array}{cc}0 & 0 \\ 0 & F^{\prime}(t)\end{array}\right), \lim _{t \rightarrow \infty} t^{-1} \int_{0}^{t} F^{\prime}(\tau) d \tau=-1$, ot lo système (3) a la forme: $x_{1}^{\prime}=0, x_{2}^{\prime}=g(t) x_{1}+F(t) x_{2}$, où $g(t)$ est une fonction bornée, telle que $\int_{0}^{\infty} g(t) d t<\infty, \lim _{t \rightarrow \infty} g(t)=0$.
HYPO'THEेSE 2.

$$
\begin{gathered}
f(t, 0)=0, \quad \| f\left(t, x_{1}\right)-f\left(t, x_{2}\|\leqslant g(t)\| x_{1}-x_{2} \|,\right. \\
\int_{0}^{\infty} g(t) d t<\infty .
\end{gathered}
$$

HYPothìse 3. L'une des conditions suivantes subsiste:

a. La norme de la matrice $Y(t) Y^{-1}(\tau)$ est bornée pour tous les $t$ et $\tau \leqslant t$, c'est-à-dire il existe un nombre $N<\infty$ tel que

$$
\left\|Y(t) Y^{-1}(\tau)\right\| \leqslant N \quad \text { lorsque } \quad \tau \leqslant t,
$$

ou bien

b. la fonction $f(t, x)$ est telle que

$$
f(t, Y(t) z)=Y(t) f(t, z)
$$

pour tout ti et le vecteur $z$ arbitraire.

Winograd a démontré son théorème en appliquant la méthode des approximations successives à l'équation intégrale

$$
x(t)=Y(t)\left(c_{0}+\int_{0}^{t} \mid Y^{-1}(\tau) f[\tau, x(\tau)] d \tau\right)
$$

dans laquelle $c_{0}=Y^{-1}(0) x(0)$ est un vecteur constant.

Nous allons démontrer que l'Hypothèse 2 de R. E. Winograd jeut être remplacée par l'hypothèse (2) plus faible, dans laquelle la méthode des approximations successives ne réussit pas. Plus précisement, on a le théorème suivant:

THÉORÈmE 1. Dans l'hypothèse que la fonction $f(t, x)$ soit partout continue $^{4}$ ) le système de conditions (5), (2), (7), de même que le système (5), (2) et (8) est suffisant pour que toutes les solutions de (3) soient bornées.

Démonstration. I. Admettons les hypothèses (5), (2), (7). On sait bien que chaque solution $x(t)$ du système (3) satisfait à l'équation (9), d'où il résulte, en vertu des hypothèses admises, que

$$
\|x(t)\| \leqslant M\left\|c_{0}\right\|+N \int_{0}^{t} g(\tau)\|x(\tau)\| d \tau .
$$

3) Cette hypothèse est équivalente à l'hypothèse que toutes les solutions du système (4) soient bornées.

4) L'hypothèse de la continuité peut être remplacée par l'hypothèse plus générale que l'équation différentielle (3) avec la condition $x(0)=Y(0) c_{0}$ soit équivalente à l'équation intégrale (9). 
On obtient facilement de la dernière inégalité (cf. [3], p. 19, lemme $1^{5}$ ))

$$
\|x(t)\| \leqslant M\left\|e_{0}\right\| \exp \left[N \int_{0}^{t} g(\tau) d \tau\right] \quad \text { pour } \quad t \geqslant 0 .
$$

L'inégalité (10) met en évidence que $x(t)$ est bornée, car il a été supposé que l'intégrale $\int_{0}^{\infty} g(t) d t$ converge (cf. (2)).

II. Admettons les hypothèses (5), (2), (8) et considérons la transformation

$$
x=Y(t) z .
$$

On vérifie facilement que la nouvelle variable $z$ satisfait à l'équation $z^{\prime}=Y^{-1} f(t, Y z)$ qui, en vertu de $1^{\prime} h y p o t h e ̀ s e ~(8)$, se réduit à

$$
z^{\prime}=f(t, z) \text {. }
$$

Le théorème de Weyl pouvant être appliqué au système (12) $(A=0)$, on conclut, que chaque solution $z$ de ce système est bornée, donc, en vertu de (11) et de (5), chaque solution $x(t)$ du système (3) a la même propriété.

\section{Travaux cités} p. $7-12$.

1] H. Weyl, Comment on the preceding paper, Amer. J. of Math. (88 (1946),

[2] Р. Э. Виноград, Об ограниченности решений правильны:х систем оияіяе. ренциальных уравнений с малььми добавками, Усігехи математичесіих паун 8.1 (1953), p. 115-120

[3] В. В. Немыцки й В. В. Степанов, Kaиecmoenthas meopust duffiepen. чиальных уравненици, Москва-Ленинград 1949.

INSTYTUT MATEMATYOZNY POLSKIEJ AKADEMII NAUK

INSTITUT MATHEMATIQUE DE L'ACADLMIE POLONAISE DISS SCIINNUAS

5) Lemme 1, publié dans [3] concerne le cas $\left\|c_{0}\right\| \neq 0$. En appliquant un passage à la limite convenable, on voit facilement que l'inégalité (10) est aussi juste au cas $c_{0}=0$.

\section{Sur la limitation et l'unicité des solutions d'un système non-linéaire d'équations paraboliques aux dérivées partielles du second ordre}

\author{
par J. SzaRSKI (Kraków)
}

Le but de la note présente est d'évaluer la différence entre deux solutions de certains problèmes aux limites relatifs au système d'équations aux dérivées partielles du second ordre de la forme

$$
\begin{gathered}
\frac{\partial z_{i}}{\partial t}=f_{i}\left(t, x_{1}, \ldots, x_{n}, z_{1}, \ldots, z_{m}, \frac{\partial z_{i}}{\partial x_{1}}, \ldots, \frac{\partial z_{i}}{\partial x_{n}}, \ldots, \frac{\partial^{2} z_{i}}{\partial x_{j} \partial x_{k}}, \ldots\right) \\
(i=1,2, \ldots, m)
\end{gathered}
$$

où le second membre de la $i$-ème équation ne dépend pas des dérivées des fonctions $z_{1}, \ldots, z_{i-1}, z_{i+1}, \ldots, z_{m}$.

La méthode consiste à comparer le système (0.1) avec des équations différentielles ordinaires en faisant intervenir la théorie des inégalités différentielles ordinaires.

Dans le $\$ 1$ nous commençons par démontrer deux lemmes qui ne sont que des modifications convenables de deux théorèmes dûs à Ważewski [2]. [3]. Dans le $\$ 2$ nous nous servons d'un système d'équations différentielles ordinaires et nous obtenons des évaluations qui permettent d'établir des critères d'unicité des solutions de certains problèmes aux limites. Par comparaison du système (0.1) avec une équation différentielle ordinaire nous obtenons dans le $\S 3$ des critères plus généraux dont l'un (théorème 3.1 ) constitue une généralisation du critère de Giuliano [1].

\$ 1. LEMME 1.1. Soit $G$ un ensemble ouvert et borné dans l'espace $\left(x_{1}, \ldots, x_{n}\right)$. Supposons que la fonction $\varphi(t, P)$, où $P=\left(x_{1}, \ldots, x_{n}\right)$, soit continue pour $P \in \bar{G}$ et $t$ appartenant $\grave{a}$ l'intervalle

$$
0<t<T \quad(T \leqslant \infty) .
$$

Posons

$$
M(t)=\max _{P \in \bar{G}} \varphi(t, P) .
$$

